\title{
Characterization of a Peptide Antibody Specific to the Adenylyl Cyclase-Associated Protein of Acanthamoeba castellanii
}

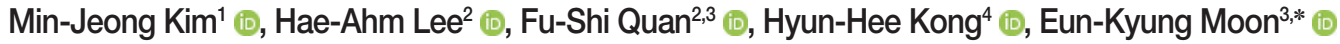

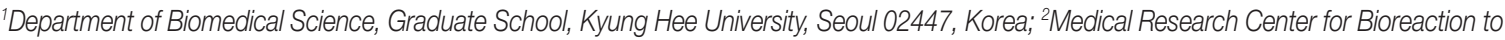 \\ Reactive Oxygen Species and Biomedical Science Institute, School of Medicine, Graduate school, Kyung Hee University, Seoul 02447, Korea; \\ ${ }^{3}$ Department of Medical Zoology, Kyung Hee University School of Medicine, Seoul 02447, Korea; ${ }^{4}$ Department of Parasitology, Dong-A University \\ College of Medicine, Busan 49201, Korea
}

\begin{abstract}
Acanthamoeba keratitis (AK) is a rare infectious disease and accurate diagnosis has remained arduous as clinical manifestations of AK were similar to keratitis of viral, bacterial, or fungal origins. In this study, we described the production of a polyclonal peptide antibody against the adenylyl cyclase-associated protein (ACAP) of $A$. castellanii, and evaluated its differential diagnostic potential. Enzyme-linked immunosorbent assay revealed high titers of $A$. castellaniispecific lgG and IgA antibodies being present in low dilutions of immunized rabbit serum. Western blot analysis revealed that the ACAP antibody specifically interacted with $A$. castellanii, while not interacting with human corneal epithelial (HCE) cells and other causes of keratitis such as Fusarium solani, Pseudomonas aeruginosa, and Staphylococcus aureus. Immunocytochemistry (ICC) results confirmed the specific detection of trophozoites and cysts of $A$. castellanii co-cultured with HCE cells. The ACAP antibody also specifically interacted with the trophozoites and cysts of 5 other Acanthamoeba species. These results indicate that the ACAP antibody of $A$. castellanii can specifically detect multiple AK-causing members belonging to the genus Acanthamoeba and may be useful for differentially diagnosing Acanthamoeba infections.
\end{abstract}

Key words: Acanthamoeba, diagnosis, adenylyl cyclase-associated protein

\section{INTRODUCTION}

Acanthamoeba keratitis (AK) is a rare infectious disease affecting the eye that can lead to permanent vision loss if not treated properly [1]. To prevent such disastrous consequences from occurring, a rapid and accurate AK diagnosis is crucial. In AK patients, bacterial or fungal coinfections are common. Members of the genera Staphylococcus and Fusarium were reported as the most common pathogens of bacterial and fungal origins encountered by AK patients, respectively [2,3]. Since symptoms of AK can be quite similar to those of bacterial and fungal keratitises, it is often misdiagnosed [4]. Therefore, the possibility of these pathogens coinhabiting the cornea along with Acanthamoeba spp. should always be considered to deliver an accurate diagnosis and appropriate treatments.

- Received 26 November 2021, revised 2 February 2022, accepted 10 February 2022.

*Corresponding author (ekmoon@khu.ac.kr)

(c) 2022, Korean Society for Parasitology and Tropical Medicine

This is an Open Access article distributed under the terms of the Creative Commons Attribution Non-Commercial License (https://creativecommons.org/licenses/by-nc/4.0) which permits unrestricted non-commercial use, distribution, and reproduction in any

medium, provided the original work is properly cited.
Currently, most cases of AK have been diagnosed by confocal microscopy, corneal scrapings, Acanthamoeba culture on agar plates, polymerase chain reaction amplification, and histopathological examinations $[5,6]$. Immunoassays using specific antibodies are also important techniques for the detection of Acanthamoeba. Four monoclonal antibodies (AMEC1, AMEC3, MTAC1, and MTAC3) generated by mixtures of A. castellanii trophozoites and cysts, a monoclonal antibody against a CPA2 transporter of A. castellanii, and a monoclonal antibody against a mannose-binding protein of A. culbertsoni have been produced to support AK diagnosis [7-9]. Polyclonal antibodies against inosine-uridine preferring nucleoside hydrolase (IPNH) and chorismate mutase (CM) of A. castellanii have also been produced for specific detection of Acanthamoe$b a[10,11]$. Despite the ongoing development of diagnostic techniques, the methods are not yet widely used. Therefore, sensitive and rapid differential diagnostics are still needed to effectively diagnose and treat AK.

Adenylyl cyclase-associated protein (ACAP) was first identified in Saccharomyces cerevisiae, and CAP (adenylyl cyclase associated protein) homolog was isolated from Dictyostelium dis- 
coideum $[12,13]$. Some of the major functions of this protein involve remodeling of the actin cytoskeleton and regulating cell motility [14]. CAP homologs exist in all species, including bacteria, yeasts, fungi, plants, and mammals [15]. Many studies have shown that CAPs are involved in various cellular and pathological processes by regulating the actin cytoskeleton and the Ras/CAMP pathway [16]. In mammals, 2 CAP isoforms have been identified. CAP1 is expressed in almost all tissues and cells, whereas CAP2 has specific expressions in skeletal and cardiac muscles, brains, and skin [14]. CAP2 is a biomarker with diagnostic and prognostic values in human gastric and breast cancer $[17,18]$. CAP2 is also a potential diagnostic biomarker for early hepatocellular carcinoma [19]. In recent years, many studies have been conducted on CAP as a biomarker for the diagnosis of human diseases. However, studies reporting the application of CAP to detect parasitic organisms are extremely limited.

In our previous study, we found that the gene expression of ACAP of A. castellanii is upregulated to a greater extent in the pathogenic strain than in the non-pathogenic strain [20]. Based on this observation, we hypothesized that ACAP could be used to specifically detect pathogenic strains of Acanthamoe$b a$. Thus, we produced a polyclonal peptide antibody targeting the ACAP of pathogenic A. castellanii, and evaluated its diagnostic potential. The objective of this study was to describe the production of ACAP antibodies and their specificities to several pathogenic Acanthamoeba spp. using enzyme-linked immunosorbent assay (ELISA), western blot analysis, and immunocytochemistry (ICC) assay.

\section{MATERIALS AND METHODS}

\section{Cell cultures}

Human corneal epithelial (HCE) cells (PCS-700-010) and Acanthamoeba castellanii Castellani (ATCC 30868) were purchased from the American Type Culture Collection. A. castellanii Neff, A. hatchetii, A. polyphaga, A. culbertsoni, and A. royreba were kindly provided by Prof. Ho-Joon Shin at Ajou University (Suwon, Korea). Fusarium solani, Pseudomonas aeruginosa, and Staphylococcus aureus were obtained from the Korea Centers for Disease Control \& Prevention (NCCP 32678, NCCP 16091, and NCCP 15920). HCE cells were cultured at $37^{\circ} \mathrm{C}$ with $5 \% \mathrm{CO}_{2}$ in endothelial cell growth medium kits (KGM BulletKit) (Lonza, Portsmouth, New Hampshire, USA). Acanthamoeba trophozoites were cultured in Peptone-Yeast-Glucose (PYG) media at $25^{\circ} \mathrm{C}$ for 5 days, and cysts were induced in encystment media $\left(95 \mathrm{mM} \mathrm{NaCl}, 5 \mathrm{mM} \mathrm{KCl}, 8 \mathrm{mM} \mathrm{MgSO}{ }_{4}, 0.4 \mathrm{mM} \mathrm{CaCl}_{2}, 1\right.$ $\mathrm{mM} \mathrm{NaHCO}_{3}$, and $20 \mathrm{mM}$ Tris-HCl, pH 9.0) at $25^{\circ} \mathrm{C}$. F. solani was cultured using Sabouraud Dextrose (SD) media at $25^{\circ} \mathrm{C}$, and P. aeruginosa and S. aureus were cultured on Brain Heart Infusion (BHI) media at $37^{\circ} \mathrm{C}$ incubators.

\section{Peptide antibody production of adenylyl cyclase- associated protein}

The peptide sequence of adenylyl cyclase-associated protein used as the immunogen is as follows: $\mathrm{NH}_{2}$-QRELLYKASQSKKPDATTFG-C-COOH. Synthesized peptide and the antibody raised against the peptide were purchased from AbFRONTIER (AbFRONTIER, Seoul, Korea). Briefly, 2 NZW rabbits were injected intradermally with $1.0 \mathrm{mg}$ of peptide-KLH conjugates with complete Freund's adjuvant on days 0 , and $0.5 \mathrm{mg}$ of the peptide with incomplete Freund's adjuvant on days 28, 42, and 56. The animals were bled on days 35 and 49 . After the 3rd immunization, the antisera titer was assessed using an indirect ELISA with the peptide-BSA conjugates as coating antigens until the titer plateaued. After the last immunization, blood samples were acquired by cardiac puncture from each rabbit.

\section{Enzyme-linked immunosorbent assay (ELISA)}

Acanthamoeba-specific antibody responses against Acanthamoeba cell lysates $\left(10^{2}\right.$ to $\left.10^{-3} \mu \mathrm{g} / \mu \mathrm{l}\right)$ from serially diluted sera (1:5 to $1: 50,000)$ were determined using ELISA as described previously [11]. Briefly, 96-well plates were coated with antigens in carbonate coating buffer overnight at $4^{\circ} \mathrm{C}$. Plates were washed 3 times with PBST and blocked with $0.2 \%$ gelatin for $2 \mathrm{hr}$ at $37^{\circ} \mathrm{C}$. Sera diluted in PBS were inoculated into wells and incubated at $37^{\circ} \mathrm{C}$ for $1 \mathrm{hr}$. Horseradish peroxidase (HRP)conjugated anti-rabbit IgG or IgA (Sigma-Aldrich, St. Louis, Missouri, USA) was added at 1:1,000 dilution in PBS and incubated at $37^{\circ} \mathrm{C}$ for $1 \mathrm{hr}$. O-phenylenediamine substrate (Zymed, San Francisco, California, USA) was dissolved in citrate-phosphate buffer with $\mathrm{H}_{2} \mathrm{O}_{2}$. The optical density values at $450 \mathrm{~nm}$ were read using EZ Read 400 microplate reader (Biochrom Ltd., Cambridge, UK). The sera of the unimmunized mice were used as a negative control.

\section{Western blot}

To verify the specificity of the ACAP antibody, western blotting was conducted using HCE cells, A. castellanii, F. solani, S. 
aureus, and P. aeruginosa [11]. Briefly, each cell lysates were confirmed on 10\% SDS-PAGE and transferred to a nitrocellulose membrane. The membrane was blocked with 5\% skim milk in TBST for $2 \mathrm{hr}$ and incubated overnight at $4^{\circ} \mathrm{C}$ with the ACAP antibody $(1: 1,000)$. The membrane was washed with TBST and probed with HRP-conjugated anti-rabbit IgG (Sigma-Aldrich) $(1: 5,000)$ for $1 \mathrm{hr}$ at RT. Bands were developed in the darkroom using Clarity Enhanced Chemiluminescence reagent (Thermo Fisher, Waltham, Massachusetts, USA) on x-ray films.

\section{Immunocytochemistry (ICC)}

To confirm the specificity of the ACAP antibody, an immunocytochemistry assay was conducted using HCE cells, Acanthamoeba spp. trophozoites, and cysts [11]. HCE cells $\left(3 \times 10^{5}\right.$ cells) were cultured on sterile cover glass in a 6 -well plate. The following day, they were co-cultured with Acanthamoeba trophozoites $\left(5 \times 10^{5}\right.$ cells $)$, and cysts $\left(5 \times 10^{5}\right.$ cells $)$ for $5 \mathrm{hr}$ at $37^{\circ} \mathrm{C}$ with $5 \% \mathrm{CO}_{2}$. After washing with PBS, the cells were fixed with ice-cold $100 \%$ methanol for 5 min at RT. Cells were washed and permeabilized with PBST for $10 \mathrm{~min}$ at RT. The cells were washed and blocked using blocking buffer (1\% bovine serum albumin and $22.52 \mathrm{mg} / \mathrm{ml}$ glycine in PBST) for 30 min at RT. The cells were incubated overnight at $4^{\circ} \mathrm{C}$ with ACAP antibody (1:200) in blocking buffer and probed with
CruzFluor $^{\mathrm{TM}} 555$ (CFL 555)-conjugated anti-rabbit IgG (1: 400; Santa Cruz, Dallas, Texas, USA) for 1 hr at RT. After washing, cells were stained with VECTASHIELD mounting medium (Abcam, Burlingame, California, USA) and observed under a fluorescent microscope (Leica DMi8, Wetzlar, Germany).

\section{Statistical analysis}

Student's $t$-test was performed using GraphPad Prism version 5 (San Diego, California, USA). Data are expressed as mean \pm SD. Statistical significance between the means of groups was denoted using an asterisk. $P$ values less than 0.05 was considered statistically significant $\left({ }^{*} P<0.05,{ }^{* *} P<0.01\right.$, and ${ }^{* * *} P<$ $0.001)$.

\section{RESULTS}

\section{Sequence analysis of ACAP of $A$. castellanii for antigen preparation}

The adenylyl cyclase-associated protein (ACAP) of A. castellanii consists of 1,458 bp and encodes 485 amino acids with a calculated mass of $53.35 \mathrm{kDa}$ (GeneBank: MW683237.1). To design a peptide antigen with optimal antigenicity, amino acid sequences of ACAP of A. castellanii were compared with those of Homo sapiens (ACAP1 and ACAP2), F. oxysporum, S. aureus, and $P$. aeruginosa (Fig. 1). Based on the secondary structure
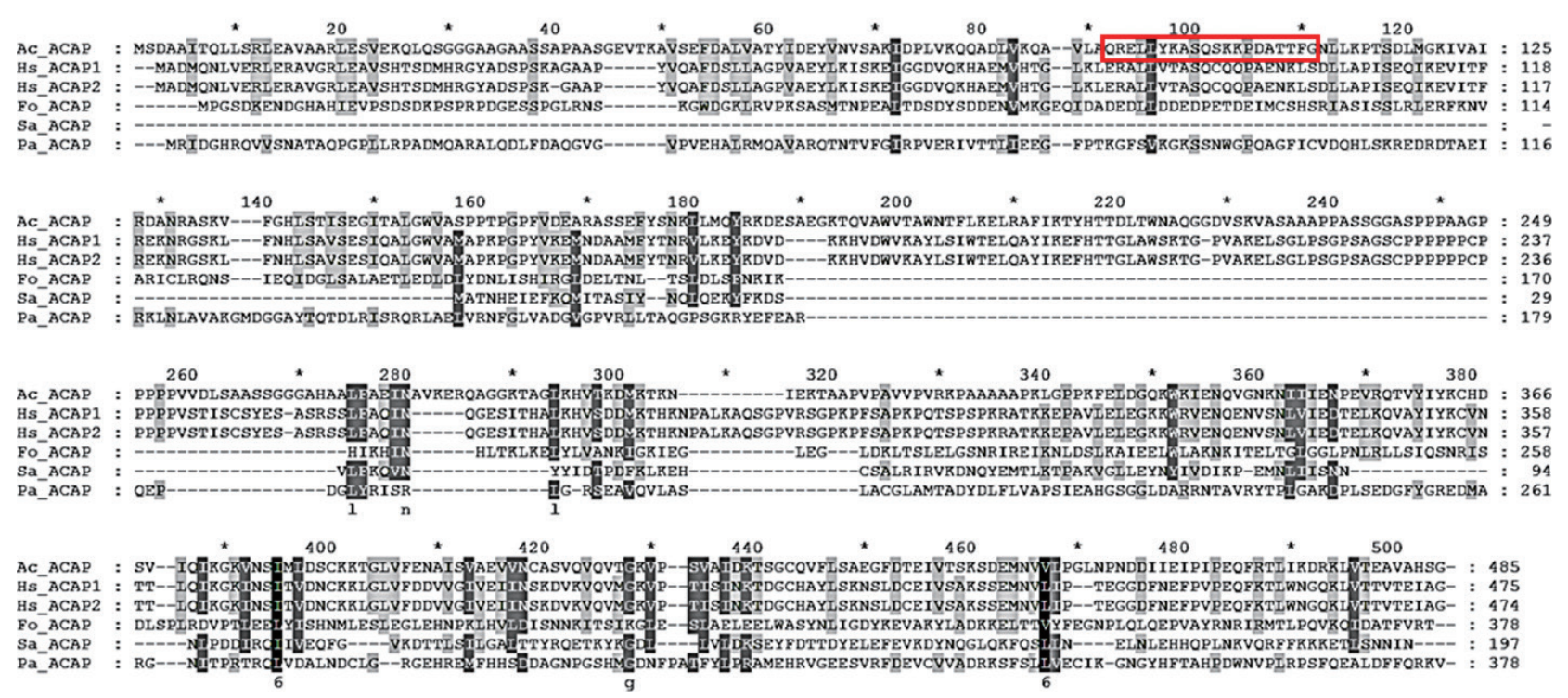

Fig. 1. Multiple sequence alignment of ACAPs. Aligned amino acid sequences of ACAP from $A$. castellanii (Ac_ACAP), H. sapiens (Hs_ ACAP1 and Hs_ACAP2), F. oxysporum (Fo_ACAP), S. aureus (Sa_ACAP), and P. aeruginosa (Pa_ACAP) were compared. The deduced amino acid sequences were aligned by using ClustalW, and the degree of conservation is represented by different shading. The boxed sequence was used to raise the anti-ACAP polyclonal antibody. 
analysis, hydrophobicity, and amino acid homology search, amino acids at positions 90-109 were selected for peptide antibody production (boxed area in Fig. 1). Amino acid sequence homology results showed that ACAP of A. castellanii showed $40.6 \%, 40.2 \%, 15.5 \%, 24.3 \%$, and $19.2 \%$ similarity with those of H. sapiens (ACAP1 and ACAP2), F. oxysporum, S. aure$u s$, and $P$. aeruginosa, each respectively (Table 1 ).

\section{Antibody responses to ACAP antigen}

ELISA was performed to titrate A. castellanii-specific IgG and IgA antibodies in low dilutions of immunized rabbit serum (Fig. 2). Acanthamoeba-specific IgG and IgA antibody responses

Table 1. Identities of ACAP amino acids as determined by BLASTP

\begin{tabular}{llcc}
\hline Name & Accession No. & Length (aa) & Identities (\%) \\
\hline Ac_ACAP & QVH35979.1 & 485 & 100 \\
Hs_ACAP1 & NP_006358.2 & 475 & 40.6 \\
Hs_ACAP2 & NP_001317431.2 & 474 & 40.2 \\
Fo_ACAP & EWY80337.1 & 378 & 15.5 \\
Sa_ACAP & CRL33834.1 & 197 & 24.3 \\
Pa_ACAP & OHQ71188.1 & 378 & 19.2 \\
\hline
\end{tabular}
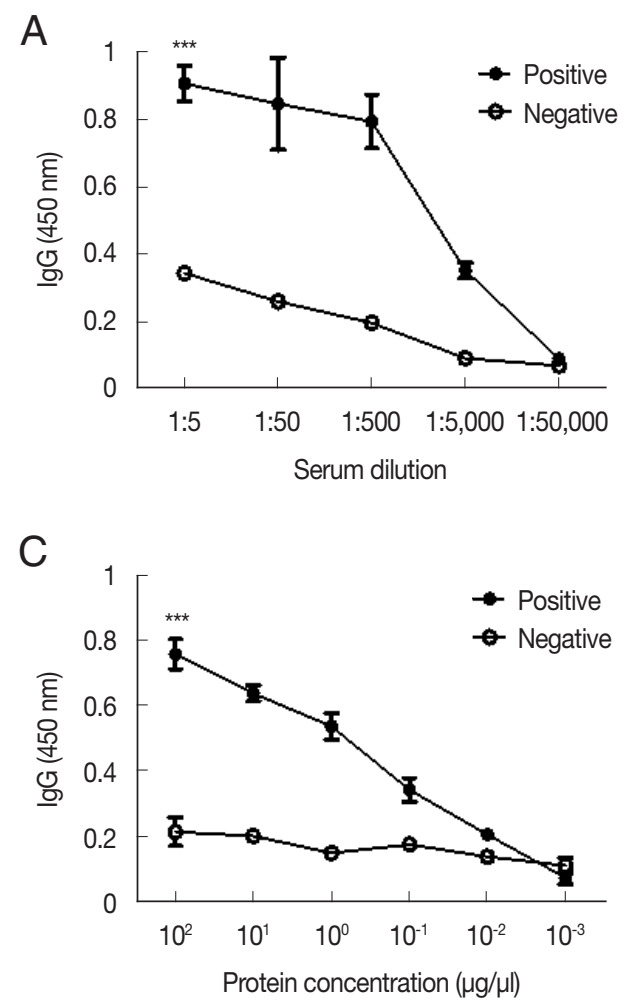

could be observed until 1:5,000 and 1:50 dilutions of immune sera, respectively (Fig. 2A, B). To establish the sensitivity of the ACAP antibody, antibodies were reacted with the serially diluted cell lysates of $A$. castellanii. The detection ranges for the Acanthamoeba-specific IgG and IgA were as low as $10^{-2} \mu \mathrm{g} / \mu \mathrm{l}$ and $10^{\circ} \mathrm{\mu g} / \mathrm{\mu l}$ of cell lysates, respectively (Fig. 2C, D). These results indicated that a high level of anti-Acanthamoeba IgG antibody and a low level of anti-Acanthamoeba IgA antibody responses could be elicited by the A. castellanii ACAP immune sera.

\section{Specificity of ACAP peptide polyclonal antibody}

To investigate the specificity of the anti-ACAP peptide polyclonal antibody, western blot analysis was performed using cell lysates of HCE cells, A. castellanii, F. solani, S. aureus, and $P$. aeruginosa (Fig. 3). The ACAP antibody reacted with A. castellanii, while such strong interactions were not observed from HCE cells, F. solani, S. aureus, and P. aeruginosa. The estimated sizes of the ACAP of HCE cells, A. castellanii, F. oxysporum, $S$. aureus, and $P$. aeruginosa were $52.25 \mathrm{kDa}, 53.35 \mathrm{kDa}, 41.58$
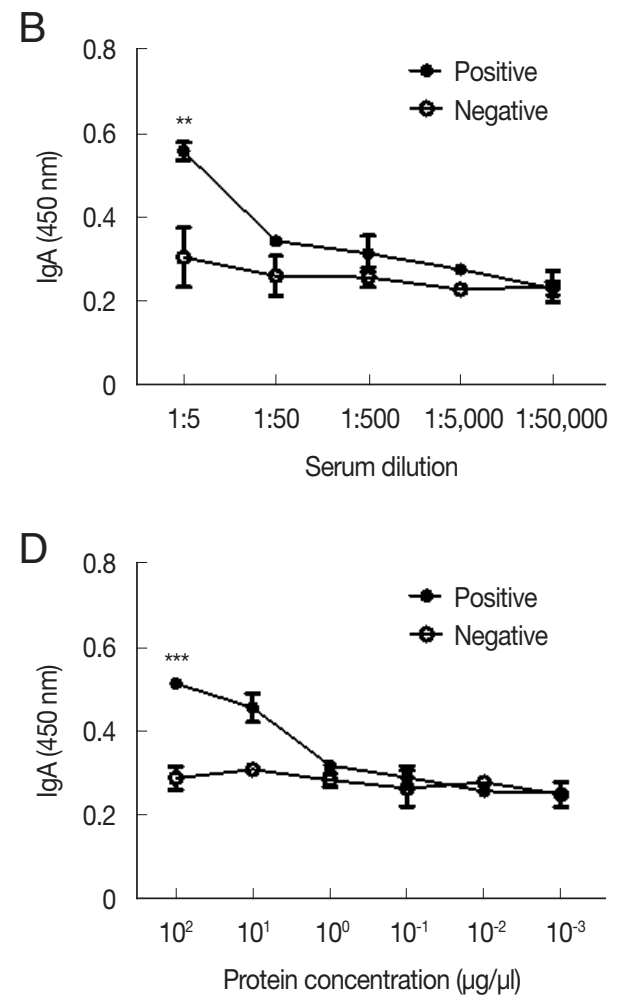

Fig. 2. Antibody responses in rabbit antiserum to ACAP of $A$. castellanii. The ACAP-specific $\lg$ G and lgA antibody titers were measured by ELISA (A, B). Sera collected from the ACAP immunized rabbits were diluted at a ratio of 1:50,000. Sensitivities of these antibodies were assayed by serially diluted cell lysates of $A$. castellanii $(C, D)$. Asterisks denote statistically significant differences between positive (•) and negative $(O)$ serum $\left({ }^{\star \star} P<0.01\right.$ and $\left.{ }^{\star \star \star} P<0.001\right)$. 
$\mathrm{kDa}, 21.67 \mathrm{kDa}, 41.58 \mathrm{kDa}$, each respectively (Table 1). Some faint bands appeared when F. solani, S. aureus, and P. aeruginosa lysates were probed with the ACAP antibody, but size differences indicate that they were of non-specific origin.

To confirm the specificity of ACAP antibody to A. castellanii trophozoites and cysts, immunocytochemistry was performed using HCE cells co-cultured with Acanthamoeba (Fig. 4). The nuclei of HCE cells were stained with DAPI (blue), but HCE cells remained unstained with ACAP antibody labeled with CFL fluorophore (red). However, A. castellanii trophozoites

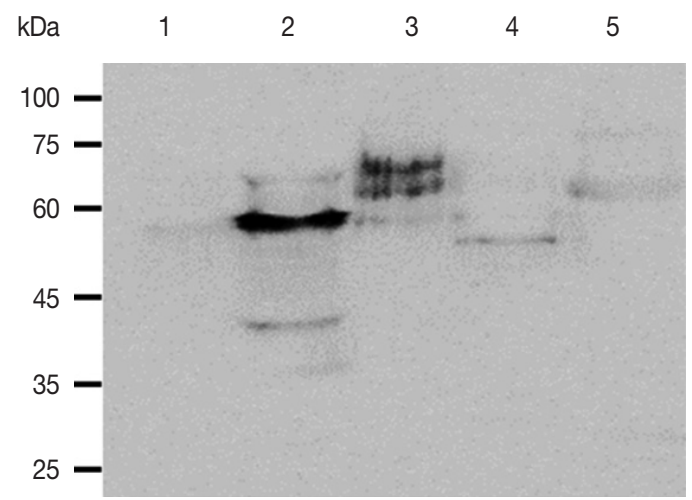

Fig. 3. Specificity of the anti-ACAP antibody. Antibody specificity was determined by western blot analysis using cell lysates from different organisms. Lane 1; HCE cells, Lane 2; A. castellanii, Lane 3; F. solani, Lane 4; S. aureus, Lane 5; P. aeruginosa. (top row) and cysts (bottom row) showed strong interaction with the ACAP antibody (red). DAPI-stained nuclei of Acanthamoeba were faintly visible because the size of Acanthamoeba nuclei is much smaller than that of HCE cells. This result demonstrated that the ACAP peptide antibody of A. castellanii specifically detected the trophozoites and cysts of A. castellanii.

\section{The specificity of ACAP Antibody to other Acanthamoeba spp.}

For further verification of the specificity of the ACAP antibody, immunocytochemistry was performed using 5 different species of Acanthamoeba. (Table 2; Fig. 5). The trophozoites and cysts of A. castellanii Neff, A. hatchetti, A. polyphaga, A. culbertsoni, and A. royreba interacted with ACAP antibodies. However, the cysts of $A$. polyphaga did not specifically react with the

Table 2. Specific detection of 5 reference Acanthamoeba spp. by immunocytochemistry

\begin{tabular}{lccc}
\hline Species & $\begin{array}{c}\text { Pussard-Pons } \\
\text { group }\end{array}$ & Trophozoites & Cysts \\
\hline A. castellanii Neff & $\|$ & + & + \\
A. hatchetti & $\|$ & + & + \\
A. polyphaga & $\|$ & + & \pm \\
A. culbertsoni & $\|$ & + & + \\
A. royreba & $\|$ & + & + \\
\hline
\end{tabular}
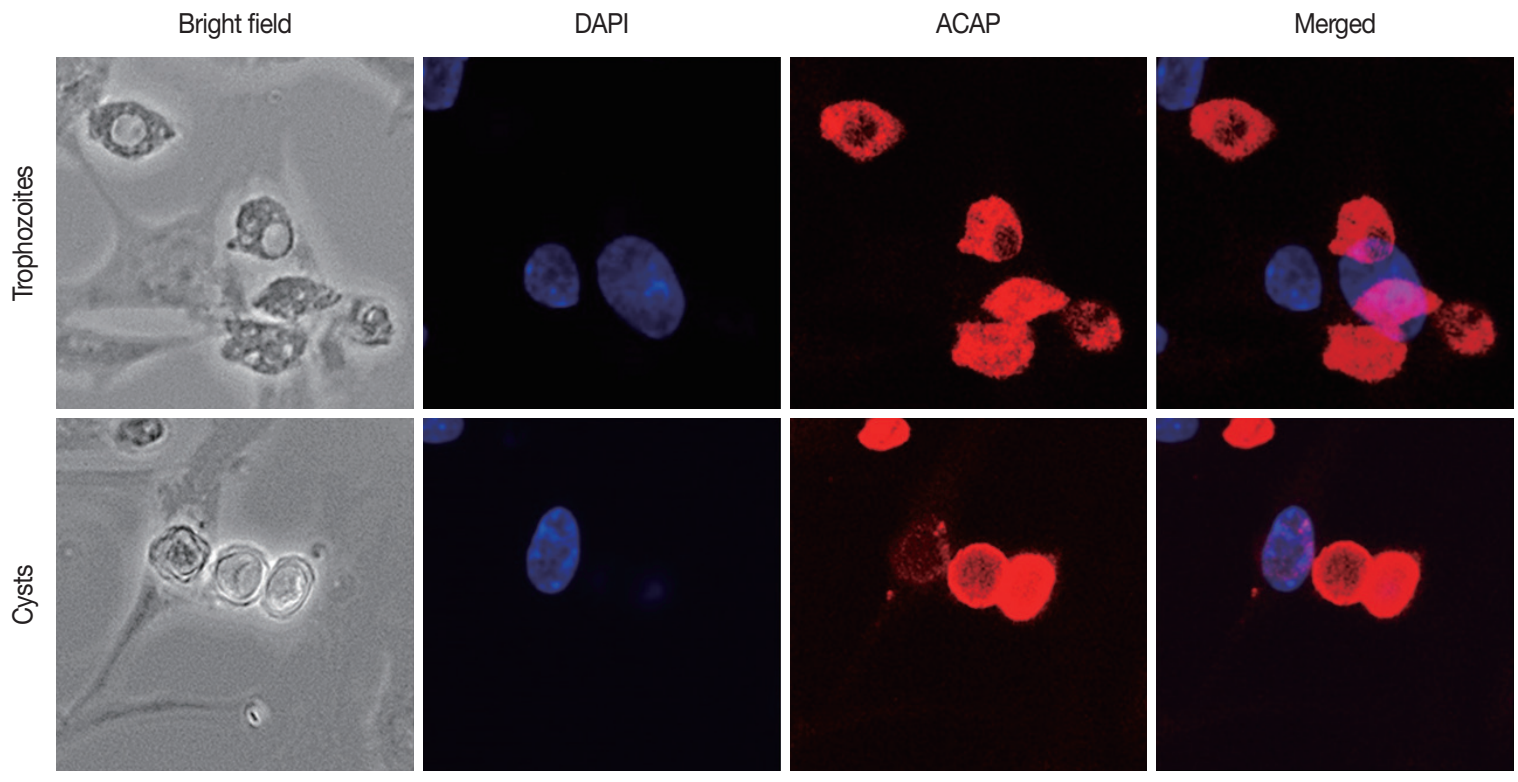

$400 \times$

Fig. 4. Immunocytochemistry assay of anti-ACAP antibody. HCE cells and A. castellanii trophozoites and cysts were co-cultured and observed under a fluorescent microscope. Bright-field, DAPI staining (blue), ACAP antibody combined with CFL-conjugated secondary antibody (red), and merged images were acquired. 


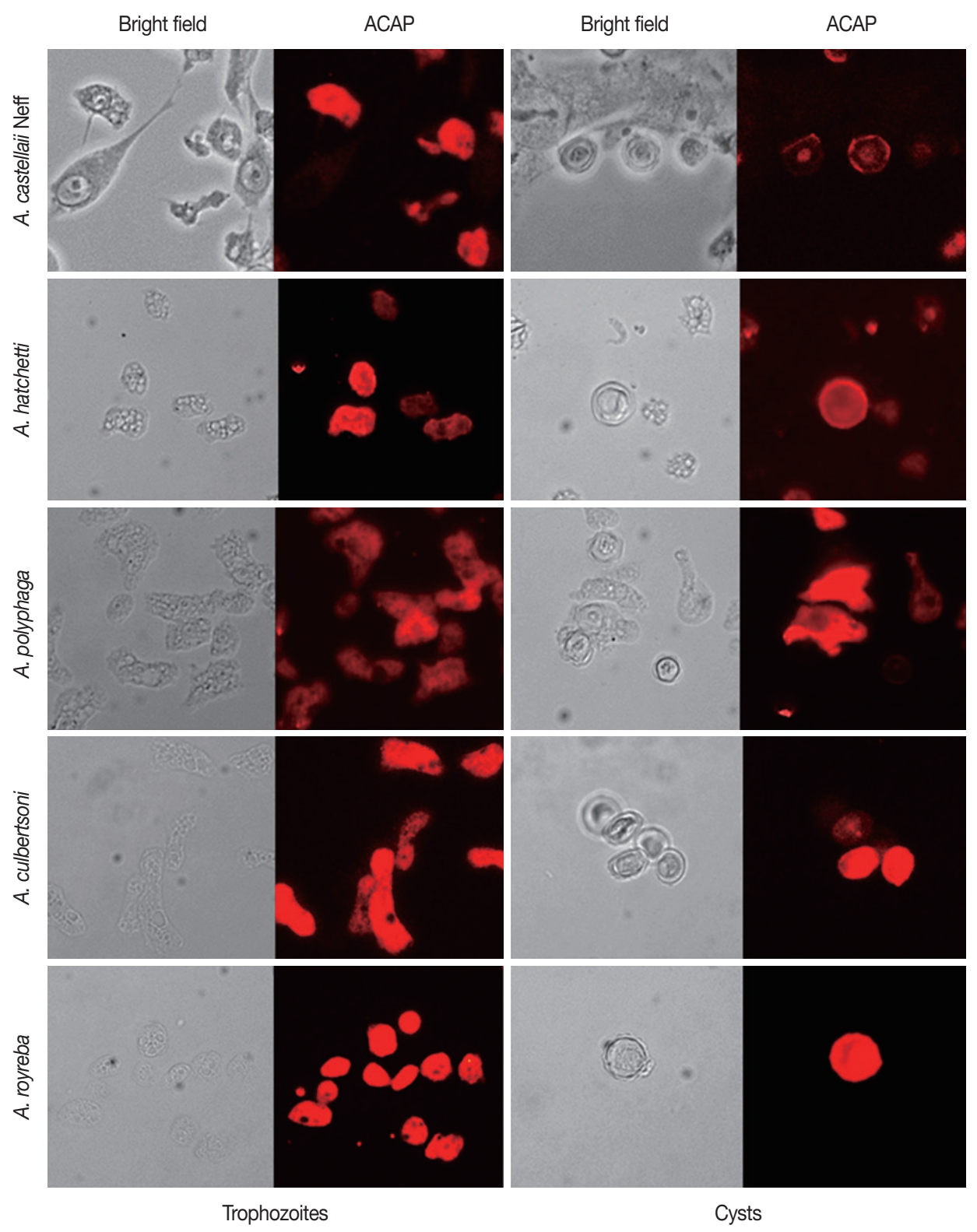

Fig. 5. Immunocytochemistry of 5 references Acanthamoeba spp. The interaction of ACAP antibody (combined with CFL-conjugated secondary antibody) with trophozoites and cysts of $A$. castellaii Neff, $A$. hatchetti, $A$. polyphaga, $A$. culbertsoni, and $A$. royreba were acquired at $400 \times$ magnification.

ACAP antibody. This result showed that the ACAP antibody of A. castellanii was capable of detecting various species of Acanthamoeba trophozoites and cysts.

\section{DISCUSSION}

For effective treatment of $\mathrm{AK}$, an accurate diagnosis in the early stages of infection is essential. Accordingly, sensitive and rapid differential diagnostic methods for AK have been stud- ied. Currently, several studies investigating the diagnostic potential of antibody-based methods for AK have been reported [7-11]. In this study, we produced a polyclonal peptide antibody against the ACAP of A. castellanii and studied its specificity. This antibody was highly specific to 6 different species of Acanthamoeba.

Acanthamoeba exists either as an infective trophozoite form or a dormant cyst form. It is thought that trophozoite-derived proteases contribute to penetration and degradation of the 
stroma, and the severe pain associated with AK [21]. Encystation prolongs the infection and renders the Acanthamoeba highly resilient to therapeutic agents [22]. It has been reported that Acanthamoeba cysts are capable of surviving over 24 years [5]. Currently, the Gram stain and Giemsa stain are widely used for the rapid detection of Acanthamoeba cysts in corneal scrapings [23]. However, false-negative and false-positive results can occur if the Acanthamoeba spp. cannot be differentiated from surrounding tissue cells and other coinfecting pathogens [23]. We have previously reported the Acanthamoeba-specific antibodies targeting the IPNH and CM of A. castellanii $[10,11]$. Although these studies revealed that these antibodies could specifically identify trophozoites of Acanthamoeba spp. from other causes of keratitis, our previous studies did not investigate their diagnostic potential against cysts of Acanthamoe$b a$ spp. The ACAP participates in the activation of adenylyl cyclase, which is upregulated and induces prespore differentiation in Dictyostelium slugs $[14,26]$. It is presumed that the ACAP is also involved in the cyst formation of Acanthamoeba. The ACAP antibody described in this study strongly reacted with both trophozoites and cysts of the aforementioned 6 Acanthamoeba spp (Figs. 4, 5). Therefore, the presence of Acanthanthamoeba trophozoites and cysts could be easily confirmed by using this antibody.

Acanthamoeba spp. is distributed in natural environments. It has been reported that $50 \%$ to $100 \%$ of healthy people possess Acanthamoeba-specific antibodies $[24,25]$. However, AK patients have significantly higher anti-Acanthamoeba IgG antibody titers than healthy people [25]. In the present study, an immunized rabbit with ACAP of A. castellanii produced Acanthamoeba-specific high IgG but low IgA (Fig. 2). Acanthamoebaspecific IgG and IgA were demonstrated to be sensitive with a detection limit as low as $10 \mathrm{ng} / \mu \mathrm{l}$ and $1 \mu \mathrm{g} / \mu \mathrm{l}$ of Acanthamoeba cell lysate, respectively (Figs. 2C, D). Therefore, if Acanthamoeba is present in corneal scrapings or ocular secretions of AK patients, it is believed that Acanthamoeba can be sufficiently detected with this antibody.

Over 20 species of Acanthamoeba have been identified, of which, 8 species (A. castellanii, A. polyphaga, A. royreba, A. culbertsoni, A. hachetti, A. griffin, A. quina, and A. lugdunensis) could cause keratitis, with A. castellanii and A. polyphaga being the most common causes of AK [22]. In the present study, we used 6 species of Acanthamoeba (A. castellanii Castellani, A. castellanii Neff, A. hatchetti, A. polyphaga, A. culbertsoni, and A. royreba). Immunocytochemistry results revealed that the ACAP anti- body reacted with all these Acanthamoeba spp (Figs. 4, 5). It would be nice to add the results of detecting A. griffin, A. qui$n a$, and A. lugdunensis by ACAP antibody. In addition, it is needed that the AK mouse model or human corneal scrapes can be used to detect Acanthamoeba spp. in future studies.

In summary, we generated a polyclonal peptide antibody against the ACAP protein of A. castellanii that specifically detected 6 species of Acanthamoeba trophozoites and cysts. This result suggests its potential for developing a diagnostic method for AK.

\section{ACKNOWLEDGMENT}

This work was supported by the National Research Foundation of Korea (NRF) grant funded by Korea government (MIST) (No. 2020R1A2C1005345).

\section{CONFLICT OF INTEREST}

The authors declare no conflict of interest related to this study.

\section{REFERENCES}

1. Page MA, Mathers WD. Acanthamoeba keratitis: a 12-year experience covering a wide spectrum of presentations, diagnoses, and outcomes. J Ophthalmol 2013; 2013: 670242. https://doi.org/ $10.1155 / 2013 / 670242$

2. Singh A, Sahu SK, Sharma S, Das S. Acanthamoeba keratitis versus mixed Acanthamoeba and bacterial keratitis: comparison of clinical and microbiological profiles. Cornea 2020; 39: 1112-1116. https:// doi.org/10.1097/ICO.0000000000002337

3. Raghavan A, Baidwal S, Venkatapathy N, Rammohan R. The Acanthamoeba-fungal keratitis study. Am J Ophthalmol 2019; 201: 31-36 https://doi.org/10.1016/j.ajo.2019.01.024

4. Fanselow N, Sirajuddin N, Yin XT, Huang AJW, Stuart PM. Acanthamoeba keratitis, pathology, diagnosis and treatment. Pathogens 2021; 10: 323. https://doi.org/10.3390/pathogens10030323

5. Szentmáry N, Daas L, Shi L, Laurik KL, Lepper S, Milioti G, Seitz B. Acanthamoeba keratitis-Clinical signs, differential diagnosis and treatment. J Curr Ophthalmol 2018; 31: 16-23. https://doi.org/10. 1016/j.joco.2018.09.008

6. Sharma S, Athmanathan S, Ata-Ur-Rasheed M, Garg P, Rao GN. Evaluation of immunoperoxidase staining technique in the diagnosis of Acanthamoeba keratitis. Indian J Ophthalmol 2001; 49: 181-186.

7. Turner ML, Cockerell EJ, Brereton HM, Badenoch PR, Tea M, Coster DJ, Williams KA. Antigens of selected Acanthamoeba species detected with monoclonal antibodies. Int J Parasitol 2005; 
35: 981-990. https://doi.org/10.1016/j.ijpara.2005.03.015

8. Weber-Lima MM, Prado-Costa B, Becker-Finco A, Costa AO, Billilad P, Furst C, de Moura JF, Alvarenga LM. Acanthamoeba spp. monoclonal antibody against a CPA2 transporter: a promising molecular tool for acanthamoebiasis diagnosis and encystment study. Parasitology 2020; 147: 1678-1688. https://doi.org/10. 1017/S0031182020001778

9. Kang AY, Park AY, Shin HJ, Khan NA, Maciver SK, Jung SY. Production of a monoclonal antibody against a mannose-binding protein of Acanthamoeba culbertsoni and its localization. Exp Parasitol 2018; 192: 19-24. https://doi.org/10.1016/j.exppara.2018.07.009

10. Park SM, Lee HA, Chu KB, Quan FS, Kim SJ, Moon EK. Production of a polyclonal antibody against inosine-uridine preferring nucleoside hydrolase of Acanthamoeba castellanii and its access to diagnosis of Acanthamoeba keratitis. PLoS One 2020; 15: e0239867 https://doi.org/10.1371/journal.pone.0239867

11. Lee HA, Chu KB, Kim MJ, Quan FS, Kong HH, Moon EK. Chorismate mutase peptide antibody enables specific detection of Acanthamoeba. PLoS One 2021; 16: e0250342. https://doi.org/10.1371/ journal.pone.0250342

12. Field J, Nikawa J, Broek D, MacDonald B, Rodgers L, Wilson IA, Lerner RA, Wigler M. Purification of a RAS-responsive adenylyl cyclase complex from Saccharomyces cerevisiae by use of an epitope addition method. Mol Cell Biol 1988; 8: 2159-2165. https:// doi.org/10.1128/mcb.8.5.2159-2165.1988

13. Noegel AA, Rivero F, Albrecht R, Janssen KP, Köhler J, Parent CA, Schleicher M. Assessing the role of the ASP56/CAP homologue of Dictyostelium discoideum and the requirements for subcellular localization. J Cell Sci 1999; 112: 3195-3203.

14. Kakurina GV, Kolegova ES, Kondakova IV. Adenylyl cyclase-associated protein 1: structure, regulation, and participation in cellular processes. Biochemistry (Mosc) 2018; 83: 45-53. https://doi. org/10.1134/S0006297918010066

15. Hubberstey AV, Mottillo EP. Cyclase-associated proteins: CAPacity for linking signal transduction and actin polymerization. FASEB J 2002; 16: 487-499. https://doi.org/10.1096/fj.01-0659rev

16. Zhang H, Ghai P, Wu H, Wang C, Field J, Zhou GL. Mammalian adenylyl cyclase-associated protein 1 (CAP1) regulates cofilin function, the actin cytoskeleton, and cell adhesion. J Biol Chem 2013; 288: 20966-20977. https://doi.org/10.1074/jbc.M113.484535
17. Li L, Fu LQ, Wang HJ, Wang YY. CAP2 is a valuable biomarker for diagnosis and prognostic in patients with gastric cancer. Pathol Oncol Res 2020; 26: 273-279. https://doi.org/10.1007/s12253018-0450-4

18. Xu L, Peng S, Huang Q, Liu Y, Jiang H, Li X, Wang J. Expression status of cyclase-associated protein 2 as a prognostic marker for human breast cancer. Oncol Rep 2016; 36: 1981-1988. https:// doi.org/10.3892/or.2016.5051

19. Fu J, Li M, Wu DC, Liu LL, Chen SL, Yun JP. Increased Expression of CAP2 Indicates Poor Prognosis in Hepatocellular Carcinoma. Transl Oncol 2015; 8: 400-406. https://doi.org/10.1016/ j.tranon.2015.08.003

20. Moon EK, Choi HS, Park SM, Kong HH, Quan FS. Comparison of proteins secreted into extracellular space of pathogenic and non-pathogenic Acanthamoeba castellanii. Korean J Parasitol 2018; 56: 553-558. https://doi.org/10.3347/kjp.2018.56.6.553

21. Clarke DW, Niederkorn JY. The pathophysiology of Acanthamoeba keratitis. Trends Parasitol 2006; 22: 175-180. https://doi. org/10.1016/j.pt.2006.02.004

22. Maycock NJ, Jayaswal R. Update on Acanthamoeba keratitis: diagnosis, treatment, and outcomes. Cornea 2016; 35: 713-720. https://doi.org/10.1097/ICO.0000000000000804

23. Sharma S, Garg P, Rao GN. Patient characteristics, diagnosis, and treatment of non-contact lens related Acanthamoeba keratitis. Br J Ophthalmol 2000; 84: 1103-1108. https://doi.org/10.1136/ bjo.84.10.1103

24. Alizadeh H, Apte S, El-Agha MS, Li L, Hurt M, Howard K, Cavanagh HD, McCulley JP, Niederkorn JY. Tear IgA and serum IgG antibodies against Acanthamoeba in patients with Acanthamoeba keratitis. Cornea 2001; 20: 622-627. https://doi.org/10.1097/00003226200108000-00013

25. Walochnik J, Obwaller A, Haller-Schober EM, Aspöck H. AntiAcanthamoeba IgG, IgM, and IgA immunoreactivities in correlation to strain pathogenicity. Parasitol Res 2001; 87: 651-656. https://doi.org/10.1007/s004360100412

26. Alvarez-Curto E, Saran S, Meima M, Zobel J, Scott C, Schaap P. cAMP production by adenylyl cyclase $\mathrm{G}$ induces prespore differentiation in Dictyostelium slugs. Development 2007; 134: 95966. https://doi.org/10.1242/dev.02775 